\title{
Robustness of System Equivalent Reduction Expansion Process on Spacecraft Structure Model Validation
}

\author{
K. K. Sairajan* and G. S. Aglietti $i^{\ddagger}$ \\ ity of Southampton, Southampton S017 1BJ, United Kingdom
}

DOI: $10.2514 / 1 . J 051476$

\begin{abstract}
Test-analysis models are used in the validation of the finite element models of spacecraft structures. Here, a probabilistic approach is used to assess the robustness of a system equivalent reduction expansion process based testanalysis model when experimental and analytical modes contain different levels of inaccuracy. The approach is applied to three spacecraft models, and Monte Carlo simulations were used to determine the sensitivity of the normalized cross-orthogonality check to the system equivalent reduction expansion process reduced matrix. The effect of parameters used in this reduction and the amount of inaccuracies that can be tolerated in the modes before failing the normalized cross-orthogonality check were also determined. The results show that the probability to pass the normalized cross-orthogonality check is highly determined by the number of modes used in the reduction. The relation between capability of the finite element models to predict the frequency-response function and the quality of the model validation determined using normalized cross-orthogonality check is also investigated, and it is observed that the quantities are not always correlated. This study also shows that the sensor locations can be optimally chosen using the system equivalent reduction expansion process reduced mass matrix, and this can increase the probability to pass the normalized cross-orthogonality check.
\end{abstract}

\section{Nomenclature}

$\boldsymbol{E}$
$f$
$i$
$H$
$\boldsymbol{J}$
$\boldsymbol{K}$
$\boldsymbol{M}$
$\boldsymbol{M}_{\mathrm{SEREP}}$
$\otimes$
$P$
$\boldsymbol{R}$
$r$
$x$
$\ddot{x}$
$\boldsymbol{\Phi}_{\mathrm{FEM}}$
$\boldsymbol{\Phi}_{\mathrm{EXP}}$
$\boldsymbol{\Phi}_{\mathrm{EXP}}^{E}$
$\boldsymbol{\Phi}_{\mathrm{FEM}}^{E}$
$\eta$
$\Omega$
$\omega$
$\zeta$

$=$
$=$
$=$
$=$
$=$
$=$
$=$
$=$
$=$
$=$
$=$
$=$
$=$
$=$

inaccuracy
applied-force vector
imaginary unit $(\sqrt{(-1)})$
frequency response
unit-square matrix
stiffness matrix
mass matrix
reduced-mass matrix using
element-by-element matrix-m
percentage of inaccuracy
square array of uniformly dist
numbers
number of modes used for res
physical-displacement vector
acceleration vector
FE modal matrix
experimental modal matrix
inaccurate experimental modal
inaccurate FE modal matrix
modal-displacement vector
forcing frequency
circular frequency
modal-damping coefficient
deleted degrees of freedom
mode number
total degrees of freedom
active degrees of freedom

$$
\begin{array}{lll}
p & = & \text { output location } \\
q & = & \text { input location } \\
\text { Superscripts } & \\
E & =\text { erroneous parameter } \\
g & =\text { generalized inverse } \\
T & =\text { matrix transpose } \\
-1 & =\text { matrix inverse }
\end{array}
$$

\section{Introduction}

$\mathbf{F}^{1}$ INITE element models (FEMs) are used to predict the structural dynamic characteristics and to verify the design of spacecraft structures. The accuracy of such models is crucial, as they are used in the coupled load analysis that is performed to determine the loads on the spacecraft during launch. Hence, inaccuracies in the model may result in inaccurate load predictions and ultimately catastrophic failures. This issue is compounded by the fact that the design margins of the spacecraft are less than those for more conventional structures to enhance the structural efficiency. It is therefore essential to verify and validate spacecraft FEMs. Finite element (FE)-predicted dynamic characteristics are verified by comparing them with experimental values obtained from structural dynamic tests. Resonance frequency values obtained from the dynamic test can be directly compared to the FE predictions, whereas mode-shape comparisons require a vector-correlation method such as modal assurance criterion (MAC) [1] or normalized cross-orthogonality (NCO) check [2]. The latter includes the mass matrix, which provides a more enhanced correlation measurement. If the test-analysis correlation indicator (MAC or NCO), falls below specified limits, the FE model should be updated to improve the match with the experimentally measured parameters.

Generally, the number of degrees of freedom (DOF) in the FEMs of a spacecraft will be in the order of a few hundred thousand and up to a few million. The dynamic test should be conducted with a few hundred accelerometers (i.e., a few hundred DOF) due to the complexity of the measurement system and sufficiency of a few important modes, called target modes, to represent the spacecraft dynamics with a required accuracy. To correlate the experimental modes with the FE modes due to the mismatch in the number of DOF, either the test results should be expanded or the FE modes should be reduced to the test locations. The first approach requires an algorithm that uses the FEM to expand the test data, and because the accuracy of 
the FEM has not yet been proved, the expanded mode shapes could be distorted by the errors in the FEM [3]. In the second approach, a reduced test-analysis model (TAM) is generated and used for the comparison with experimental results.

Various reduction methods have been developed for generating the TAM. The most widely used reduction method known as static reduction was suggested by Guyan [4]. As the name indicates, this procedure gives an exact reduction for a static problem, but it fails to accurately represent a dynamic system. This is because the inertia terms of the reduced DOF are ignored in the formulation of the projection matrix. Irons [5] also suggested a modal reduction by eliminating the DOF with negligible inertial forces. It was shown that incorporating the inertia terms ignored in the static reduction can improve the ability of the reduced system to represent the dynamic characteristics of the original system [6]. Kammer developed a reduction technique known as Modal TAM using FEM normal modes [3]. This method is suitable for the test-analysis correlation because it does not require a recomputation of TAM for each frequency, as in the case of dynamic condensation [7]. Modal TAM can exactly predict the FE modes and the frequencies of the actual system even after the reduction. The procedure uses the normal modes for the TAM generation and yields a very small matrix that enables an easy inversion. However, Modal TAM is highly sensitive if there are differences between test and analysis mode shapes. To overcome this difficulty, Kammer introduced a hybrid TAM [ 8 ] by combining the ability of the Modal TAM to accurately represent the FEM target modes with the static TAM representation for the residual modes.

An approximate reduction technique called improved reduced system (IRS) has been developed by O'Callahan [9]. This method does not require a solution of the full-system eigenvalues, but it can reduce the system with better accuracy to predict the low-frequency resonances than static reduction. Friswell et al. [10] extended the IRS method by using the dynamic reduction as the basic transformation instead of static reduction. They also introduced an iteratively generated correction term using the best available estimate of the reduced model to converge to the modal parameters of the full system. Analytical model improvement developed by Berman and Nagy [11] uses the measured real normal modes and natural frequencies to improve the mathematically determined mass and stiffness matrix of a linear system. This method can yield modes that exactly match with the experimental modes, but the procedure failed to retain the physical properties of the stiffness matrix. To overcome this difficulty, experimentally determined mode data and structural connectivity information obtained from the analytical method were used to optimally improve the deficient analytical stiffness matrix [12].

Butland and Avitabile [13] used the modal parameters obtained from the test to update the reduced-order finite-element models of two subsystems and then combined using component-mode synthesis to generate a test-updated system-level model. To avoid the modal-based correlation matrix in the mid-frequency range test-analysis verification, a frequency-response-based reduction technique was presented [14]. The principal directions were extracted directly from the analytical frequency response and used to derive the transformation matrix to reduce the impedance matrix to the sensor locations. This reduction technique is particularly suitable for systems with high-modal density in the frequency range of interest, and it avoids the difficult task of selecting the dynamically important target modes. The accuracy of the technique, however, is dependent upon the number of principal directions used in the model reduction. Bergman et al. [15] used a probabilistic investigation to quantify the effect of errors in the experimental mode shapes on the test-analysis orthogonality. They analyzed a typical spacecraft structure using static, modal, and IRS reduction methods, and then observed that the test-analysis orthogonality is highly sensitive to the experimental errors. A fixed percentage of experimental error was simulated using a simple equation, and Monte Carlo simulations were employed to estimate the probability of meeting the orthogonality criteria.

The system equivalent reduction expansion process (SEREP) [16] can also exactly reproduce the dynamic characteristics of the original system, and it was found to be very useful in the normalized crossorthogonality check between analytical and experimental mode shapes. Compared to other reduction techniques, SEREP has advantages such as the ability to perform reduction using an arbitrary selection of modes, as well as the fact that the reduced system preserves the frequency and mode shapes of the original system for selected modes of interest. Avitabile [17] described the various considerations to be taken to avoid the pitfalls in the test-analysis correlation process. A mass or stiffness matrix can be obtained from the FE method, but the mass matrix is considered more accurate, and it is typically used in the NCO. Aglietti et al. [18] analyzed the various available techniques to generate a TAM, and they identified SEREP as being the most suitable technique for the generation of a spacecraft TAM. They also investigated the effect of inaccuracies (implemented as noise) in the mode shape of a spacecraft structure on the SEREP and NCO. It has been observed that, maintaining the same target-mode shapes for the correlation, as the number modes used in the reduction process increases, there is a decrease in the probability of meeting the NCO. It was also shown that optimality criteria based on the effective-independence (EFI) matrix can be used to improve the probability of meeting the NCO. In Ref. [19], a probabilistic approach was used to assess the robustness of the $\bar{M}$ AC and NCO in the validation of FE model of a spacecraft; and it was also shown that when the modes are corrupted by noise, meeting the typical MAC requirement is much easier than meeting the NCO requirement specified by the aerospace agencies [20,21].

The present work extends the preliminary findings reported in Refs. $[18,19]$. By considering three different spacecraft models, it demonstrates that when the number of target modes is smaller than the number of sensors, it is detrimental to try to improve the quality of the reduced SEREP matrix by including more modes in the reduction process. When the number of modes used in the reduction equals the number of sensors, passing the NCO check becomes practically impossible. Most importantly, a novel contribution of this work is to use real practical examples to show that the capability of passing a typical NCO test does not necessarily imply that such a model is able to accurately predict the dynamic response of the satellite, and a model that does not pass the NCO could more accurately predict some of the responses. The other contribution of this work is to introduce a simple optimization procedure to increase the probability of success of the SEREP-based TAM in the NCO check.

In practice, inaccuracies are not only present in the experimental results, but also in the FE estimation of modal parameters, and it is therefore important to assess the effect of both of these inaccuracies on the TAM and NCO. Hence, in this work, three cases are considered: a) inaccuracies in the experimental modes, b) inaccuracies in the analytical mode shapes, and c) inaccuracies in both the experimental and the analytical modes. SEREP-based TAMs are generated for three different spacecraft structures and for a set of synthetic random modes to derive general conclusions on the robustness of SEREP-based TAMs when different percentages of inaccuracies are present in the modes. The inaccuracies are simulated using a simple error model, and Monte Carlo simulations are used to quantify the sensitivity of the quality check to the SEREP-based TAM. Effects of parameters, such as the number of modes used in the SEREP reduction, the number of target modes, and the independence between the targets modes on the NCO are evaluated for different classes of spacecraft FE models. The capability of spacecraft FE models to predict the displacement-based frequency-response function when the FE models possess different levels of probabilities of passing the NCO were also assessed. Finally, a simple optimization procedure to increase the probability of success in the NCO check using SEREP-based TAM is described.

\section{Background Theory}

To present the results of the study carried out, the fundamental theoretical approach used for the model reduction needs to be explained. The Craig-Bampton [22] method is commonly used for the dynamic reduction of spacecraft FEMs, but Craig-Bampton reduced FEMs contain both physical and modal coordinates, and 
hence they cannot be used for direct comparison with the test results, which are in the physical coordinates. A modal reduction followed by an expansion using a suitable output-projection matrix is used in SEREP $[2,16]$. This method can be used for test-analysis correlation studies.

The equation of motion for an $n$ DOF undamped system is:

$$
\boldsymbol{M}_{n, n} \ddot{x}_{n}+\boldsymbol{K}_{n, n} x_{n}=f
$$

Here, $\boldsymbol{M}_{n, n}, \boldsymbol{K}_{n, n}, x_{n}, \ddot{x}_{n}$, and $f$ are the mass matrix, stiffness matrix, displacement vector, acceleration vector, and applied-force vector respectively. The subscripts denote the dimension of the arrays. The eigensolution of this system can be approximated using $m$ modal vectors $(m<n)$. Then $x_{n}$ can be obtained as:

$$
x_{n}=\boldsymbol{\Phi}_{n, m} \eta_{m}
$$

Here, $\boldsymbol{\Phi}_{n, m}$ is the modal matrix and $\eta_{m}$ is the displacement vector in modal coordinates. The vector $x_{n}$ can be partitioned as measured or active DOF denoted as $x_{a}$ and deleted DOF as $x_{d}$. Hence:

$$
x_{a}=\boldsymbol{\Phi}_{a, m} \eta_{m}
$$

Here, $\boldsymbol{\Phi}_{a, m}$ is the reduced modal matrix in terms of active DOF, and this matrix is generally not square. If the number of modal coordinates $m$ is equal to the number of active DOF $a$ (a condition known as SEREPa [23]), the reduced modal matrix becomes square and can be inverted to obtain:

$$
\eta_{a}=\boldsymbol{\Phi}_{a, a}^{-1} x_{a}
$$

Generally, the number of active DOF in a spacecraft FEM will be much higher than the number of target modes $(a>m)$. Hence, $\boldsymbol{\Phi}_{a, m}$ will be a rectangular matrix, and a generalized inverse [24] is required to determine the $\eta_{m}$. The generalized inverse can be generated by premultiplying both sides of Eq. (3) by $\boldsymbol{\Phi}_{a, m}^{T}$ to generate a square matrix on the right-hand side of the equation; which can then be inverted and used to obtain the equation for $\eta_{m}$ as:

$$
\eta_{m}=\left(\boldsymbol{\Phi}_{a, m}^{T} \boldsymbol{\Phi}_{a, m}\right)^{-1} \boldsymbol{\Phi}_{a, m}^{T} x_{a}=\boldsymbol{\Phi}_{a, m}^{g} x_{a}
$$

where $\boldsymbol{\Phi}_{a, m}^{g}$ is the generalized inverse of the modal matrix $\boldsymbol{\Phi}_{a, m}$. Hence, the displacement of the full system in terms of the reduced displacement can be determined using Eqs. (2), (므), and (ㅁ) as:

$$
x_{n}=\boldsymbol{\Phi}_{n, m} \boldsymbol{\Phi}_{a, m}^{g} x_{a}
$$

Substituting Eq. (6) into Eq. (1) and pre-multiplying by the transpose of modal matrix and the transpose of generalized inverse of the modal matrix gives:

$$
\begin{aligned}
& \left(\boldsymbol{\Phi}_{a, m}^{g}\right)^{T} \boldsymbol{\Phi}_{n, m}^{T} \boldsymbol{M}_{n, n} \boldsymbol{\Phi}_{n, m} \boldsymbol{\Phi}_{a, m}^{g} \ddot{x}_{a}+\left(\boldsymbol{\Phi}_{a, m}^{g}\right)^{T} \boldsymbol{\Phi}_{n, m}^{T} \boldsymbol{K}_{n, n} \boldsymbol{\Phi}_{n, m} \boldsymbol{\Phi}_{a, m}^{g} x_{a} \\
& \quad=\left(\boldsymbol{\Phi}_{a, m}^{g}\right)^{T} \boldsymbol{\Phi}_{n, m}^{T} f
\end{aligned}
$$

If the mass-normalized modes are used, SEREP-reduced TAM mass matrix is given by:

$$
\boldsymbol{M}_{a, a}^{\mathrm{TAM}}=\left(\boldsymbol{\Phi}_{a, m}^{g}\right)^{T} \boldsymbol{\Phi}_{a, m}^{g}
$$

\section{Sensitivity of the Test Analysis Model}

\section{A. Finite Element Model}

FEMs of three different spacecraft were considered for the testanalysis correlations. The surface models of these spacecraft are shown in the Fig. 1. The first FEM consists of 7153 nodes and 6962 elements, and it represents the structural model of a mini-spacecraft having a mass of $75.7 \mathrm{~kg}$. The modal analysis, performed using NASTRAN [25] showed 16 normal modes of less than $150 \mathrm{~Hz}$ in the base fixed-boundary condition simulating the launch configuration. The second spacecraft model was developed by Surrey Satellite Technology Limited (SSTL), U. K. It consists of 121,285 nodes and 81,795 elements with a mass of $300 \mathrm{~kg}$. There were 24 normal modes in the based fixed condition with frequencies less than $150 \mathrm{~Hz}$. The third spacecraft model was developed by EADS Astrium Limited, U.K. for their AEOLUS spacecraft. This model consists of 95,980 nodes and 109,295 elements. The normal mode analysis in the base fixed-boundary condition showed that there were 295 modes having frequencies less than $150 \mathrm{~Hz}$. The spacecraft has a total mass of $1800 \mathrm{~kg}$. These FEMs are chosen in such a way that different classes
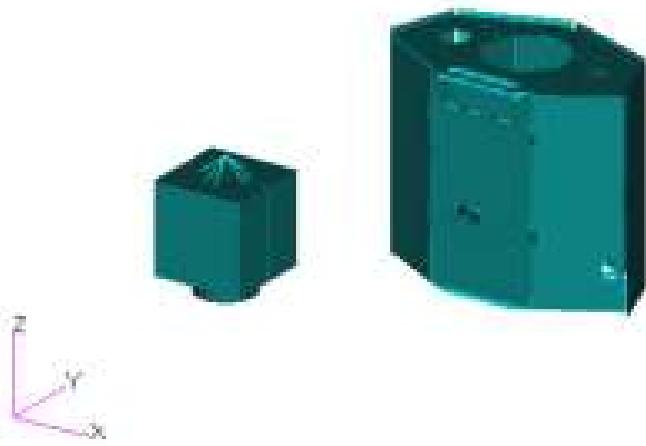

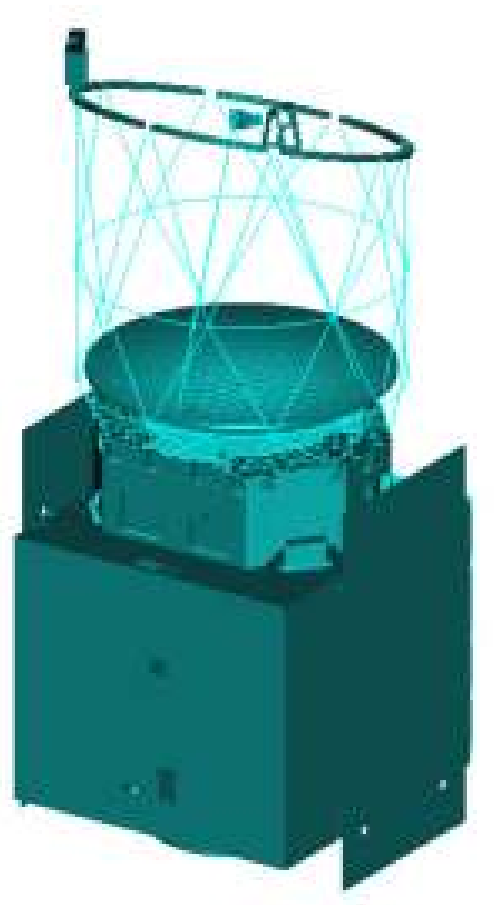

c) Large spacecraft
(EADS Astrium Limited) a) Mini spacecraft b) Medium size spacecraft (SSTL, UK)

Fig. 1 Three spacecraft structure models. 
of spacecraft are covered in this study. Figure 1 also shows the relative sizes of different spacecraft considered in this study.

\section{B. Target-Mode Selection}

Common target-mode selection criteria were applied to all the spacecraft. The target modes for the correlation studies were chosen based on the effective mass of the modes, modal-kinetic energy, and modal-strain energy [26]. For the test-analysis correlation, modes up to $150 \mathrm{~Hz}$ were considered in this study. All the modes with effective mass greater than or equal to $2 \%$ of the total mass of the spacecraft that fell within the specified frequency were chosen as target modes. In addition, modes with $50 \%$ or more modal-strain energy or modalkinetic energy of the total system and within the frequency of $150 \mathrm{~Hz}$ were also selected as target modes. This ensured that the local modes that gave peak responses in subsystems were also included in the correlation process.

\section{Monte Carlo Simulation}

The accuracy of modal parameters extracted from the dynamic test depends on several factors, such as the amount of noise in the measurements, the accuracy of the instruments, and the test setup. This leads to some inaccuracy in the estimation of the modal parameters, and the nature of these inaccuracies are complicated. FEM results also contain some inaccuracies compared to the actual system performance, mainly due to the assumptions in the idealization of the structure and limitation in the simulation of the real loading conditions. Simplified methods were used to simulate these inaccuracies and to generate the synthetic test data by different researchers $[14,18]$. In this work, a simplified multiplicative error model is used to represent the experimental and FE inaccuracies. It is defined by equation:

$$
\boldsymbol{E}=\left(\boldsymbol{J}+\frac{P}{100}(-\boldsymbol{J}+2 \boldsymbol{R})\right)
$$

Here, $\boldsymbol{J}$ is a unit-square matrix with dimensions equal to the number of DOF, $P$ is the percentage of inaccuracy, and $\boldsymbol{R}$ is a square array of uniformly distributed random numbers on the open interval $(0,1)$ with the same dimensions as $\boldsymbol{J}$. This model is simplified, but it is sufficient for the Monte Carlo simulation to assess the sensitivity of the modal reduction method to the different case of inaccuracies. The modes obtained from the FEM, $\boldsymbol{\Phi}_{\text {FEM }}$ are used to generate the synthetic experimental modes $\boldsymbol{\Phi}_{\mathrm{EXP}}^{E}[18,19]$. These synthetic modes are obtained by the Hadamard product (element-by-element matrix multiplication) of $\boldsymbol{\Phi}_{\mathrm{FEM}}$ and inaccuracy, $\boldsymbol{E}_{1}$, as:

$$
\boldsymbol{\Phi}_{\mathrm{EXP}}^{E}=\boldsymbol{\Phi}_{\mathrm{FEM}} \otimes \boldsymbol{E}_{1}
$$

Similarly, the noise-affected FE modes, $\boldsymbol{\Phi}_{\text {FEM }}^{E}$, were computed using $\boldsymbol{\Phi}_{\mathrm{FEM}}$ and inaccuracy, $\boldsymbol{E}_{2}$, generated using a different random number as given by:

$$
\boldsymbol{\Phi}_{\mathrm{FEM}}^{E}=\boldsymbol{\Phi}_{\mathrm{FEM}} \otimes \boldsymbol{E}_{2}
$$

The first mode shape of Spacecraft 3 obtained from the FE analysis is shown in Fig 2. This is a lateral mode in the $X$ direction. The dotted lines in the Fig. 2 show the undeformed structure. This analytical mode shape is determined using 33 tri-axial accelerometers, and hence each mode shape will have only 99 components or DOF. These 33 sensor locations on Spacecraft 3 are shown in Fig. 3 using two different views of the structure for the clarity of representation. The nominal first mode of Spacecraft 3 and the corresponding distorted mode shape with $25 \%$ of inaccuracy are illustrated in Fig 4 . It can be observe that the first nominal mode is predominant in the direction of $X$, and hence the amplitude is predominant for the DOFs that correspond to $1,4,7, \ldots, 97$. The SEREP mass matrix calculated using these types of modes would have 99 rows and columns irrespective of number of modes used.

Then the normalized cross-orthogonality check (also known as weighted MAC [1] ) is performed using the equation [2] :

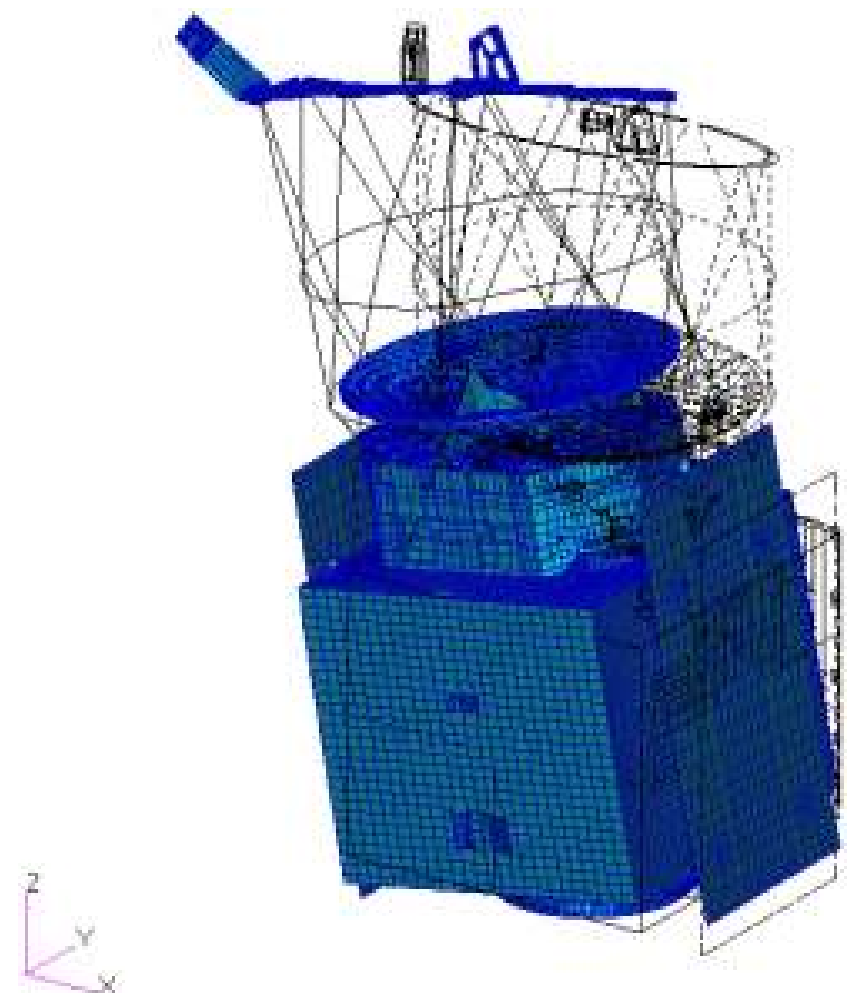

Fig. 2 The first mode shape of Spacecraft 3 at $15.96 \mathrm{~Hz}$.

$$
\boldsymbol{N} \boldsymbol{C} \boldsymbol{O}=\frac{\left(\boldsymbol{\Phi}_{\mathrm{EXP}}^{T} \boldsymbol{M}_{\mathrm{SEREP}} \boldsymbol{\Phi}_{\mathrm{FEM}}\right)^{2}}{\left(\boldsymbol{\Phi}_{\mathrm{EXP}}^{T} \boldsymbol{M}_{\mathrm{SEREP}} \boldsymbol{\Phi}_{\mathrm{EXP}}\right)\left(\boldsymbol{\Phi}_{\mathrm{FEM}}^{T} \boldsymbol{M}_{\mathrm{SEREP}} \boldsymbol{\Phi}_{\mathrm{FEM}}\right)}
$$

The NCO measures the correlation between the analytically predicted mode shapes and the mode shapes determined from the dynamic test. This enhanced version of MAC uses a mass-weighting matrix, and hence includes the mass distribution in the correlation process to give more weighting to the DOFs with more mass associated with them [1]. The off-diagonal terms in the NCO matrix help to identify whether the comparison is between the same or different mode pairs. The test-analysis correlation requirements are specified by agencies such as the National Aeronautics and Space Administration (NASA) [20] and the European Space Agency (ESA) [21]; for example, the correlation is considered to be successful if all the diagonal terms in the cross-orthogonality matrix are greater than 0.9 and all other values in the matrix are less than 0.1 .

\section{Results and Discussion}

\section{A. Effect of the Number of Modes in the System Equivalent} Reduction Expansion Process on the Normalized-Orthogonality Check

In this study, the normalized cross-orthogonality check was performed using the SEREP-reduced model of different spacecraft structure to assess the robustness of SEREP TAM on the different types of inaccuracies. Three cases were considered for each spacecraft model. In the first case, only experimental modes were assumed inaccurate, whereas in the second case, FE modes were considered inaccurate. In the third case, both experimental and FE modes were simulated with the same percentage of inaccuracies, although different random vectors were used for the generation of error. The percentage of inaccuracy was varied from 0.001 to $50 \%$ for each case, and Monte Carlo simulations were used to compute the probability of meeting the NCO criteria based on 1000 computational results per each level of inaccuracy. Here, consistent with NASA or ESA requirements, the correlation is considered to be successful if all the diagonal terms in the cross-orthogonality matrix are greater than 0.9 and all other values in the matrix are less than 0.1 . Based on this criterion, the probability of meeting the NCO for different spacecraft is shown in Tables $\underline{1}-\underline{3}$. The target modes for each 

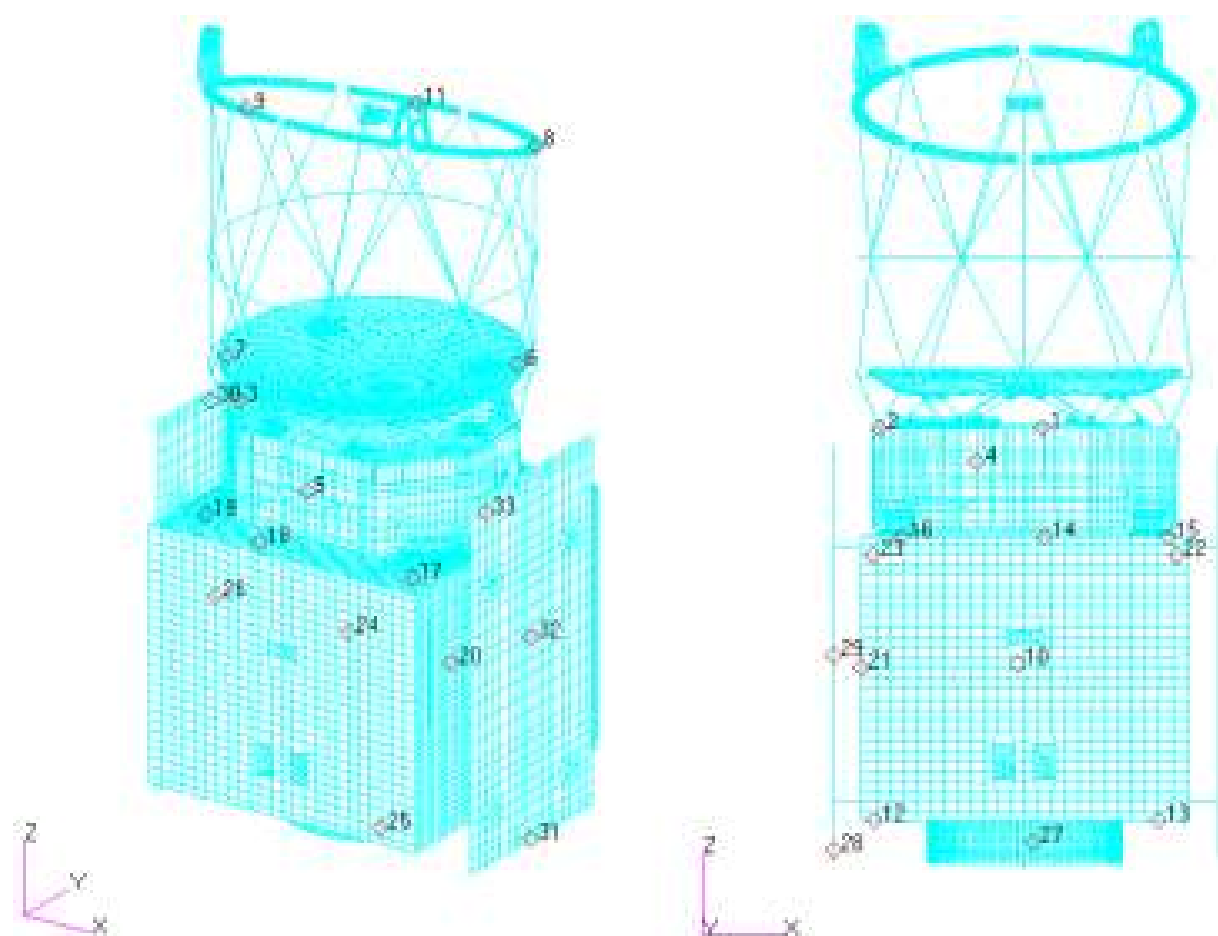

Fig. 3 Sensor locations on Spacecraft 3.

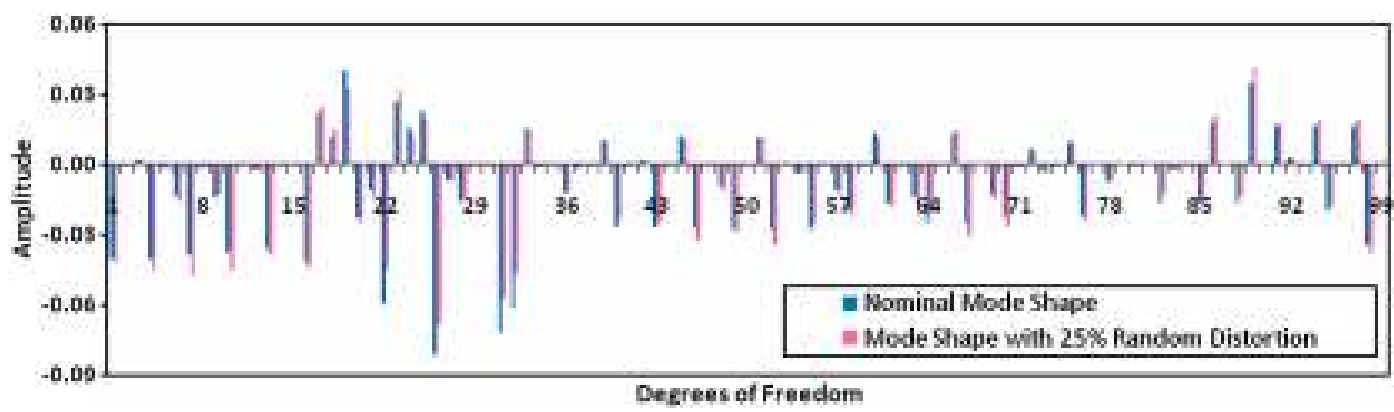

Fig. 4 Coefficients of nominal and erroneous mode shapes determined using 33 tri-axial sensors.

spacecraft were chosen based on the method described in Sec. III.B. As per this criterion, the number of target modes (NTM) was found to be 11 for Spacecraft 1 and 12 for Spacecrafts 2 and 3. To correlate these target modes, 99 DOFs that correspond to the sensor locations in the vibration test were selected $[18,19]$. Spacecraft 1 has only 11 target modes, but the same number of sensor locations (99 DOFs) is used for the comparison.
From the results in the Tables 1-3, it can be observed that for all the spacecraft models, as the number of modes used in the reduction (NMR) increase from the minimum required number, the probability of success of NCO reduces. The reduced modal matrix must contain the target mode shapes, and thus the minimum number of modes to be used in SEREP is the number of target modes. It is possible to include further mode shapes, as in the case of SEREPa. The dimensions of the

Table 1 Probability of NCO diagonal terms $>0.9$ and off-diagonal terms $<0.1$ for Spacecraft 1

\begin{tabular}{lccccccccccc}
\hline \hline NMR & \multicolumn{10}{c}{ Percentage of inaccuracy } & Case (NTM = 11) \\
\hline & 50 & 25 & 10 & 5 & 2.5 & 1 & 0.1 & 0.01 & 0.001 & \\
\hline 11 & 1.1 & 67.5 & 100.0 & $\rightarrow$ & & & & & & Error in experimental modes \\
12 & 0.5 & 58.9 & 100.0 & $\rightarrow$ & & & & & & \\
20 & $\leftarrow$ & 0.0 & 25.7 & 94.7 & 100.0 & $\rightarrow$ & & & & \\
50 & & & $\leftarrow$ & 0.0 & 3.6 & 96.8 & 100.0 & $\rightarrow$ & & \\
99 & & & & & & $\leftarrow$ & 0.0 & 90.6 & 100.0 & \\
\hline 11 & 0.0 & 20.9 & 100.0 & $\rightarrow$ & & & & & & \\
12 & 0.0 & 12.0 & 100.0 & $\rightarrow$ & & & & & & \\
20 & $\leftarrow$ & 0.0 & 2.6 & 92.1 & 100.0 & $\rightarrow$ & & & & \\
50 & & & $\leftarrow$ & 0.0 & 0.5 & 96.4 & 100.0 & $\rightarrow$ & & \\
99 & & & & & $\leftarrow$ & 0.0 & 0.4 & 91.1 & 100.0 & \\
\hline 11 & 0.0 & 6.8 & 98.3 & 100.0 & $\rightarrow$ & & & & & Error in both FE and experimental modes \\
12 & 0.0 & 2.1 & 98.3 & 100.0 & $\rightarrow$ & & & & & \\
20 & $\leftarrow$ & 0.0 & 0.6 & 56.5 & 99.9 & $\rightarrow$ & & & & \\
50 & & & & $\leftarrow$ & 0.0 & 66.8 & 100.0 & $\rightarrow$ & & \\
99 & & & & & & $\leftarrow$ & 0.0 & 72.7 & 100.0 & \\
\hline \hline
\end{tabular}


Table 2 Probability of NCO diagonal terms $>0.9$ and off-diagonal terms $<0.1$ for Spacecraft 2

\begin{tabular}{lcccccccccc}
\hline \hline NMR & \multicolumn{10}{c}{ Percentage of inaccuracy } \\
\hline & 50 & 25 & 10 & 5 & 2.5 & 1 & 0.1 & 0.01 & 0.001 & Case (NTM = 12) \\
\hline 12 & 0.0 & 0.6 & 83.7 & 100.0 & $\rightarrow$ & & & & & Error in experimental modes \\
20 & $\leftarrow$ & 0.0 & 0.4 & 34.2 & 96.6 & 100.0 & $\rightarrow$ & & & \\
50 & & & & & $\leftarrow$ & 0.0 & 100.0 & $\rightarrow$ & & \\
99 & & & & & & & & $\leftarrow$ & 0.0 & Error in FE modes \\
\hline 12 & $\leftarrow$ & 0.0 & 23.4 & 99.6 & 100.0 & $\rightarrow$ & & & & \\
20 & & $\leftarrow$ & 0.0 & 0.4 & 22.9 & 100.0 & $\rightarrow$ & & & \\
50 & & & & & $\leftarrow$ & 0.0 & 91.4 & 100.0 & $\rightarrow$ & \\
99 & & & & & & & & $\leftarrow$ & 0.0 & \\
\hline 12 & $\leftarrow$ & 0.0 & 9.0 & 96.2 & 100.0 & $\rightarrow$ & & & & Error in both FE and experimental modes \\
20 & & $\leftarrow$ & 0.0 & 0.1 & 18.1 & 99.9 & 100.0 & $\rightarrow$ & & \\
50 & & & & & $\leftarrow$ & 0.0 & 74.2 & 100.0 & $\rightarrow$ & \\
99 & & & & & & & & $\leftarrow$ & 0.0 & \\
\hline \hline
\end{tabular}

Table 3 Probability of NCO diagonal terms $>0.9$ and off-diagonal terms $<0.1$ for Spacecraft 3

\begin{tabular}{lccccccccccc}
\hline \hline NMR & \multicolumn{10}{c}{ Cercentage of inaccuracy } & \\
\hline & 50 & 25 & 10 & 5 & 2.5 & 1 & 0.1 & 0.01 & 0.001 & Case (NTM = 12) \\
\hline 12 & $\leftarrow$ & 0.0 & 9.4 & 84.7 & 100.0 & $\rightarrow$ & & & & Error in experimental modes \\
20 & & & & & $\leftarrow$ & 0.0 & 39.9 & 100.0 & $\rightarrow$ & \\
50 & & & & & & $\leftarrow$ & 0.0 & 0.2 & 100.0 & \\
99 & & & & & & & & $\leftarrow$ & 0.0 & Error in FE modes \\
\hline 12 & $\leftarrow$ & 0.0 & 2.5 & 71.7 & 100.0 & $\rightarrow$ & & & & \\
20 & & & & & $\leftarrow$ & 0.0 & 38.7 & 100.0 & $\rightarrow$ & \\
50 & & & & & & $\leftarrow$ & 0.0 & 0.1 & 100.0 & \\
99 & & & & & & & & $\leftarrow$ & 0.0 & \\
\hline 12 & $\leftarrow$ & 0.0 & 3.1 & 42.6 & 96.0 & 100.0 & $\rightarrow$ & & & Error in both FE and experimental modes \\
20 & & & & & $\leftarrow$ & 0.0 & 3.6 & 100.0 & $\rightarrow$ & \\
50 & & & & & & & $\leftarrow$ & 0.0 & 100.0 & \\
99 & & & & & & & $\leftarrow$ & 0.0 & \\
\hline \hline
\end{tabular}

different parameters used for the computation of SEREP mass matrix are:

$$
\begin{gathered}
\boldsymbol{M}_{\mathrm{SEREP}}=\left[\left[\boldsymbol{\Phi}_{\mathrm{DOF}, \mathrm{NMR}}^{T} \boldsymbol{\Phi}_{\mathrm{DOF}, \mathrm{NMR}}\right]^{-1} \boldsymbol{\Phi}_{\mathrm{DOF}, \mathrm{NMR}}^{T}\right]^{T} \\
\quad \times\left[\left[\boldsymbol{\Phi}_{\mathrm{DOF}, \mathrm{NMR}}^{T} \boldsymbol{\Phi}_{\mathrm{DOF}, \mathrm{NMR}}\right]^{-1} \boldsymbol{\Phi}_{\mathrm{DOF}, \mathrm{NMR}}^{T}\right]
\end{gathered}
$$

As per the definition, $\boldsymbol{M}_{\text {SEREP }}$ is a square matrix irrespective of the number of modes used in the reduction, and its dimension always equals the DOF or sensor locations. For the calculation of $\boldsymbol{M}_{\mathrm{SEREP}}$ for Case 1 in Table 1, nominal FE modes $\boldsymbol{\Phi}_{\text {FEM }}$ were used, whereas noise-affected FE modes $\boldsymbol{\Phi}_{\mathrm{FEM}}^{E}$ were used for the estimation of $\boldsymbol{M}_{\text {SEREP }}$ for Cases 2 and 3. When the NMR is greater than the NTM, more modes are used for the computation of the $\boldsymbol{M}_{\mathrm{SEREP}}$ than the minimum requirement. Such cases lead to a reduction in the probability of passing the NCO performed by using the reduced mass matrix due to the decrease in the diagonal values and the increase in the off-diagonal values of the cross-orthogonality matrix. The minimum number of modes required to be incorporated in the reduction is that of the target modes, whereas the maximum number of modes considered here is equal to the number of DOFs.

Tables $1-3$ also show that the inaccuracies in the FE modes have more probability to fail the NCO than the inaccuracies in the experimental modes alone. Referring to Eq. (13), the TAM mass matrix is calculated using the FE mode shapes, and hence error in the FE modes will reflect in $\boldsymbol{M}_{\mathrm{SEREP}}$, but it will be nominal when inaccuracy exists only in the experimental modes. When FE modes are corrupted, NCO is calculated by replacing $\boldsymbol{\Phi}_{\mathrm{FEM}}$ with $\boldsymbol{\Phi}_{\mathrm{FEM}}^{E}$ in Eq. (12). The combined effect of $\boldsymbol{M}_{\text {SEREP }}$ and $\boldsymbol{\Phi}_{\text {FEM }}^{E}$, which are inaccurate due to the uncorrelated and different random numbers, results in a decrease in probability of meeting the correlation criterion. When both the FE and the experimental modes contain inaccuracies, the probability of passing NCO will be further reduced due to error in all the terms in the right-hand side of Eq. (12). This is shown in Fig. $\underline{5}$ by extracting the required results $(\mathrm{NMR}=12,20$, 50 , and 99) from Tables $1-3$ for a better representation of the results.

When the number of modes used in the reduction is equal to the number of sensor locations, even a very low percentage $(0.1)$ of error leads to failure in the NCO. This is unreasonable, as in practice for such small differences between test and analysis modes, one would expect to pass a NCO. This type of reduction known as SEREPa and its probability of meeting the NCO is shown in the last row of each case in the Tables $1-3$. The SEREPa reduction is computationally efficient, as it can avoid a pseudo-inverse due to its inherent squarematrix property. Its high sensitivity to inaccuracies, however, prevents its practical use. Apart from the general features of a SEREP-reduced model in NCO, it can also be observed that Spacecraft 3 is the most vulnerable to any kind of inaccuracies, and Spacecraft 1 is least affected with inaccuracies to pass the correlation criteria. The results also show that for all three spacecraft, the SEREP should be carried out with a minimum number of modes, as increasing the number of modes sharply reduces the probability of passing the NCO even for very small percentage of inaccuracies.

\section{B. Effect of Number of the Target Modes on the Normalized Cross-Orthogonality Check}

Table 4 shows the probability of meeting the NCO when the number of target modes (NTM) is increased from 12 to 20 for Spacecraft 3 . These results are fairly obvious, but they are presented here for completeness. As expected, in all the cases as the NTM increases, the probability of successfully meeting the NCO reduces compared to the few target modes due to the increased requirement of modes in reduction. Also, more target modes must be incorporated in the cross correlation. Here a minimum of 20 target modes are required to be incorporated in the reduction, and these 20 modes are required again in the $\mathrm{NCO}$, hence the probability of success is less than the values shown in Table $\underline{3}$. Similar results were obtained for the 


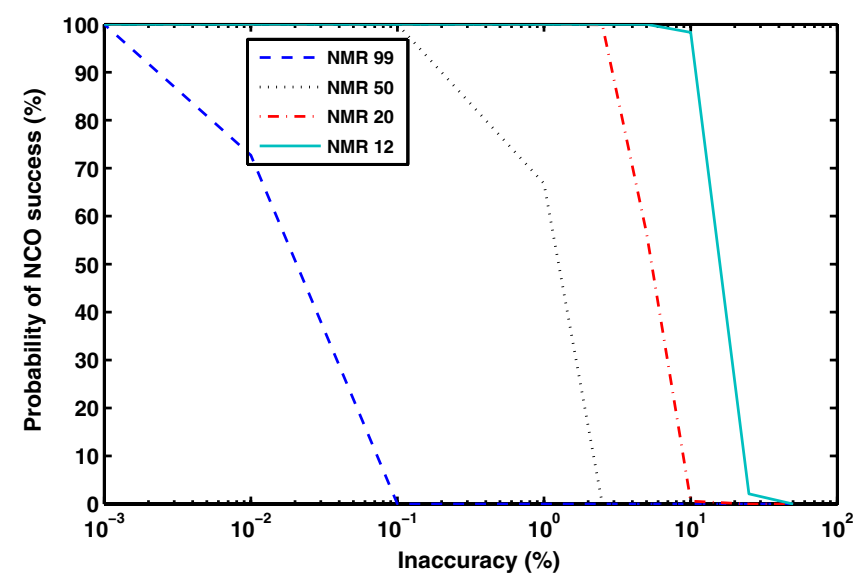

a) Spacecraft 1

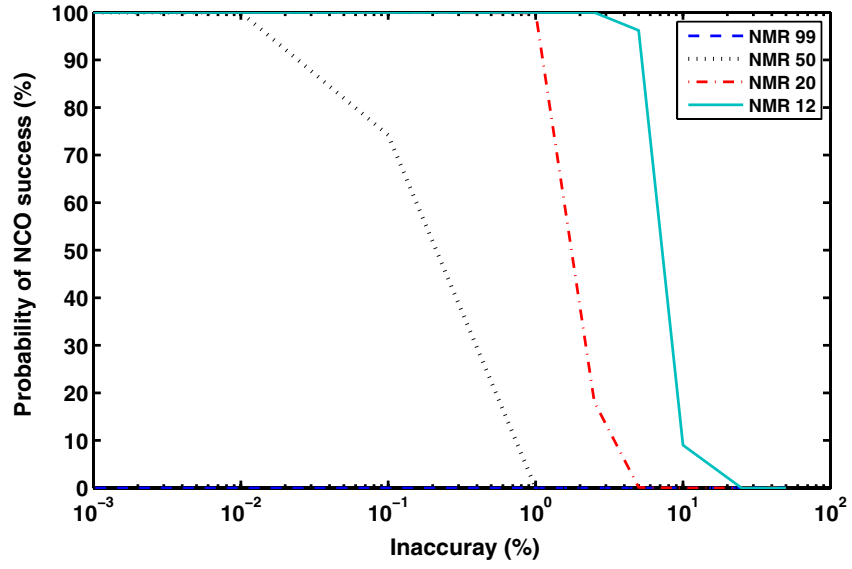

b) Spacecraft 2

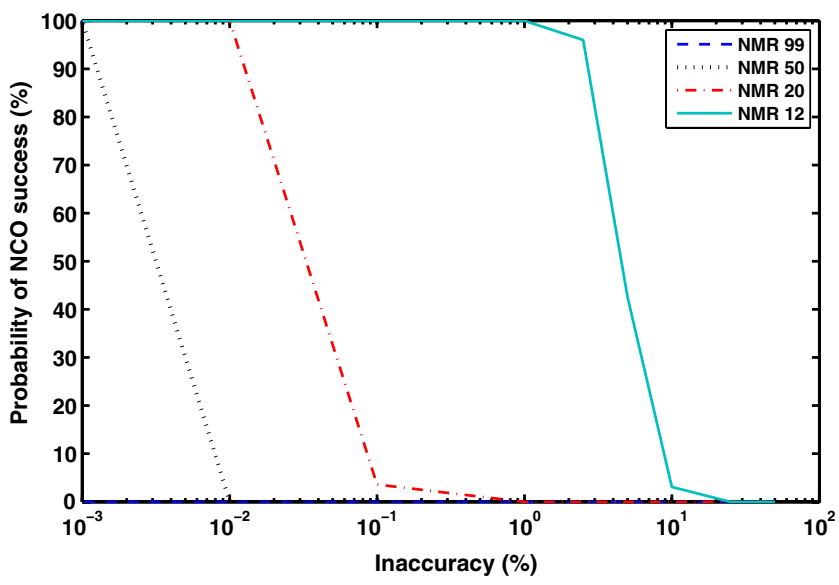

c) Spacecraft 3

Fig. 5 Probability of NCO success when inaccuracies are present in both the FE and the experimental modes.

other spacecraft models, and hence their numerical values are not reported here.

\section{Effect of Independence Between the Target Modes}

For all the spacecraft considered for correlation studies, irrespective of the percentage of inaccuracies in the mode shapes, NTM and NMR play significant roles in the normalized crossorthogonality check. It is evident that the highest probability of passing the NCO occurs when NTM = NMR. Once the target modes are selected based on the specified criteria, apart from the NTM and NMR, it was observed that independence between the target modes affects the correlation process. The effective independence matrix between the target modes was computed for all the inaccuracy levels using the Eq. (14).

$$
\boldsymbol{E} \boldsymbol{F} \boldsymbol{I}_{\mathrm{DOF}, \mathrm{DOF}}=\boldsymbol{\Phi}_{\mathrm{FEM}}\left[\boldsymbol{\Phi}_{\mathrm{FEM}}^{T} \boldsymbol{\Phi}_{\mathrm{FEM}}\right]^{-1} \boldsymbol{\Phi}_{\mathrm{FEM}}^{T}
$$

Each term in the diagonal of this matrix indicates the fractional contribution of each sensor location to the independence of the target modes [27]. The smallest value in the diagonal of the EFI matrix

Table 5 Characteristics of the target modes

\begin{tabular}{lc}
\hline \hline Structure & Standard deviation \\
\hline Synthetic & 0.04 \\
Spacecraft 1 & 0.13 \\
Spacecraft 2 & 0.14 \\
Spacecraft 3 & 0.16 \\
\hline \hline
\end{tabular}

Table 4 Probability of NCO diagonal terms $>0.9$ and off diagonal terms $<0.1$ for spacecraft 3 with NTM $=20$

\begin{tabular}{|c|c|c|c|c|c|c|c|c|c|c|}
\hline NMR & & & & Perc & entag & of in & ccura & & & Case \\
\hline & 50 & 25 & 10 & 5 & 2.5 & 1 & 0.1 & 0.01 & 0.001 & \\
\hline $\begin{array}{l}20 \\
50 \\
99\end{array}$ & & & & & $\leftarrow$ & $\begin{array}{c}0.0 \\
\leftarrow\end{array}$ & $\begin{array}{l}8.4 \\
0.0\end{array}$ & $\begin{array}{c}100.0 \\
0.2 \\
\leftarrow\end{array}$ & $\begin{array}{c}\overrightarrow{ } \\
100.0 \\
0.0\end{array}$ & Error in experimental modes \\
\hline $\begin{array}{l}20 \\
50 \\
99\end{array}$ & & & & & $\leftarrow$ & $\begin{array}{c}0.0 \\
\leftarrow\end{array}$ & $\begin{array}{c}8.1 \\
0\end{array}$ & $\begin{array}{c}100.0 \\
0.1 \\
\leftarrow\end{array}$ & $\begin{array}{c}\overrightarrow{ } \overrightarrow{00.0} \\
0.0\end{array}$ & Error in FE modes \\
\hline $\begin{array}{l}20 \\
50 \\
99\end{array}$ & & & & & $\leftarrow$ & 0.0 & $\begin{array}{c}0.4 \\
\leftarrow\end{array}$ & $\begin{array}{c}100.0 \\
0.0 \\
\leftarrow\end{array}$ & $\begin{array}{c}\overrightarrow{ } \\
100.0 \\
0.0\end{array}$ & Error in both FE and experimental modes \\
\hline
\end{tabular}


Table 6 Probability of NCO diagonal terms $>0.9$ and off-diagonal terms $<0.1$ for synthetic modes

\begin{tabular}{|c|c|c|c|c|c|c|c|c|c|c|}
\hline \multirow[t]{2}{*}{ NMR } & \multicolumn{9}{|c|}{ Percentage of inaccuracy } & \multirow[t]{2}{*}{ Case $(\mathrm{NTM}=12)$} \\
\hline & 50 & 25 & 10 & 5 & 2.5 & 1 & 0.1 & 0.01 & 0.001 & \\
\hline 12 & 100.0 & $\rightarrow$ & & & & & & & & Error in experimental modes \\
\hline 20 & 100.0 & $\rightarrow$ & & & & & & & & \\
\hline 50 & 19.5 & 100.0 & $\rightarrow$ & & & & & & & \\
\hline 99 & & & & $\leftarrow$ & 0.0 & 1.1 & 100.0 & $\rightarrow$ & & \\
\hline 12 & 100.0 & $\rightarrow$ & & & & & & & & Error in FE modes \\
\hline 20 & 100.0 & $\rightarrow$ & & & & & & & & \\
\hline 50 & 18.2 & 100.0 & $\rightarrow$ & & & & & & & \\
\hline 99 & & & & $\leftarrow$ & 0.0 & 2.7 & 100.0 & $\rightarrow$ & & \\
\hline 12 & 100.0 & $\rightarrow$ & & & & & & & & Error in both FE and experimental modes \\
\hline 20 & 100.0 & $\rightarrow$ & & & & & & & & \\
\hline 50 & 0.0 & 99.4 & 100.0 & $\rightarrow$ & & & & & & \\
\hline 99 & & & & $\leftarrow$ & 0.0 & 0.1 & 100.0 & $\rightarrow$ & & \\
\hline
\end{tabular}

corresponds to the most dependent DOF. To compare the target modes of different spacecraft, standard deviations of the corresponding diagonal values of the EFI matrix were computed. Table $\underline{5}$ shows the characteristics of different spacecraft based on the EFI of their target modes. The table also includes the characteristics of a synthetic modal matrix generated using random numbers to obtain highly uncorrelated modes. This synthetic modal matrix contained 99 modes and 99 DOFs. It indicates that target modes of Spacecraft 3 have the highest variation in the fractional components of modes compared to the other spacecraft models. The results shown in Tables proved that Spacecraft 3 has the least probability of succeeding the NCO, and Spacecraft 1 has the highest probability of succeeding for a particular percentage of error. Hence, it can be inferred that as the standard deviation of the diagonal terms of the EFI matrix increases, there will be less probability of succeeding in the normalized cross-correlation check. This is valid in the case of synthetic modes as well. Table $\underline{6}$ shows the NCO results for the synthetic modes, showing that even with $25 \%$ error in mode shapes, it can also pass the NCO for NMR $=12$. The number of target modes was taken as 12 . These synthetic modes have a much-greater probability of success than any other spacecraft structures. It can also be noted that standard deviation of the diagonal terms of the EFI matrix generated using 12 synthetic target modes is lower than any other spacecraft structure.

\section{Effect of Inaccuracy in Modal Parameters} on Frequency-Response Functions

The validation of the FEM is carried out by comparing and correlating some model parameters such as resonance frequencies and mode shapes with the experimentally retrieved parameters. This correlation exercise can be relatively sophisticated and requires a TAM, which can be obtained as described in the previous sections. What is ultimately important is the capability of the FEM to predict the dynamic response of the structure (e.g., the FRFs describing the loads in structural elements where the margins are particularly low). Hence, an accurate prediction of the frequency response using the FEM is necessary for the design of the spacecraft structure. In this section, the inaccuracies in the frequency-response functions of the different spacecraft are considered to investigate the relationship between the success in the NCO and capability to predict the dynamic response accurately. Referring to Tables 1 and 2, Spacecraft 1 can easily pass the NCO compared to Spacecraft 2 . In the case of Spacecraft 1, even if there is an inaccuracy of $10 \%$ in both FE and experimental modes, there is a $98 \%$ probability of passing the NCO, whereas Spacecraft 2 only has a $9 \%$ probability of clearing the NCO when the NMR $=$ NTM. These two spacecraft models were used to analyze their displacement frequency-response functions when modal parameters are corrupted with inaccuracies.

Mode shapes can be used to compute the displacement frequencyresponse function (FRF) using the equation [28]:

$$
H_{p q}(\Omega)=\sum_{k=1}^{r} \frac{\boldsymbol{\Phi}_{k p} \boldsymbol{\Phi}_{k q}}{\omega_{k}^{2}-\Omega^{2}+2 i \Omega \zeta_{k} \omega_{k}}
$$

Here, $\boldsymbol{\Phi}_{k p}$ is the value of the $k$ th mode at the $p$ th output location, $\boldsymbol{\Phi}_{k p}$ is the value of the $k$ th mode at the $q$ th input location, $w_{k}$ is the undamped natural frequency of the $k$ th mode in $\mathrm{rad} / \mathrm{s}, i$ is imaginary number, $\Omega$ is the excitation frequency in $\mathrm{rad} / \mathrm{s}, \zeta_{k}$ is the modal damping coefficient, and $r$ is the number of modes used for the

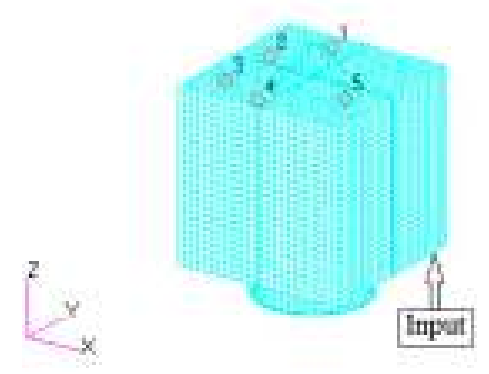

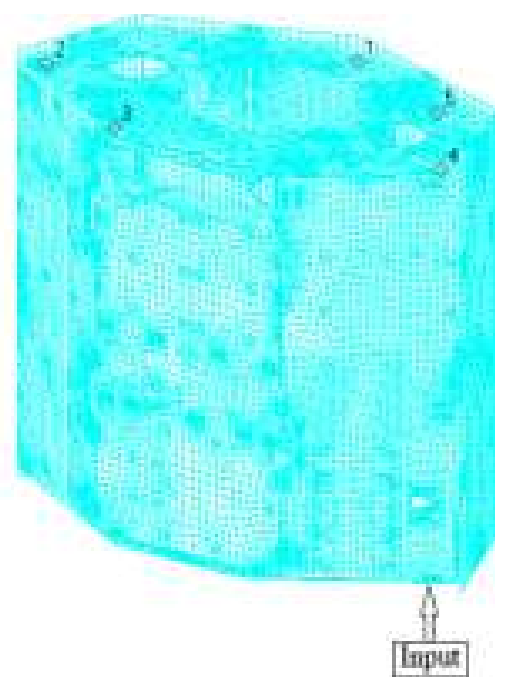

b) Spacecraft 2 


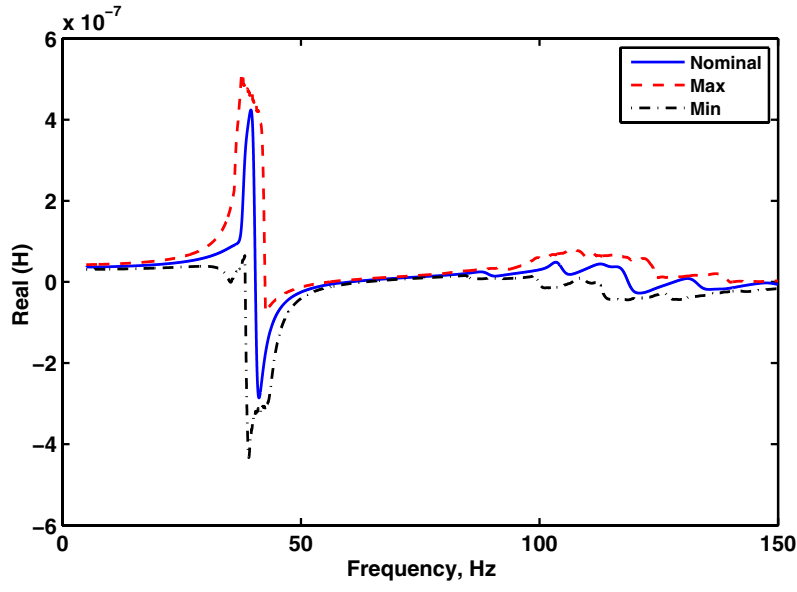

a) Location 1

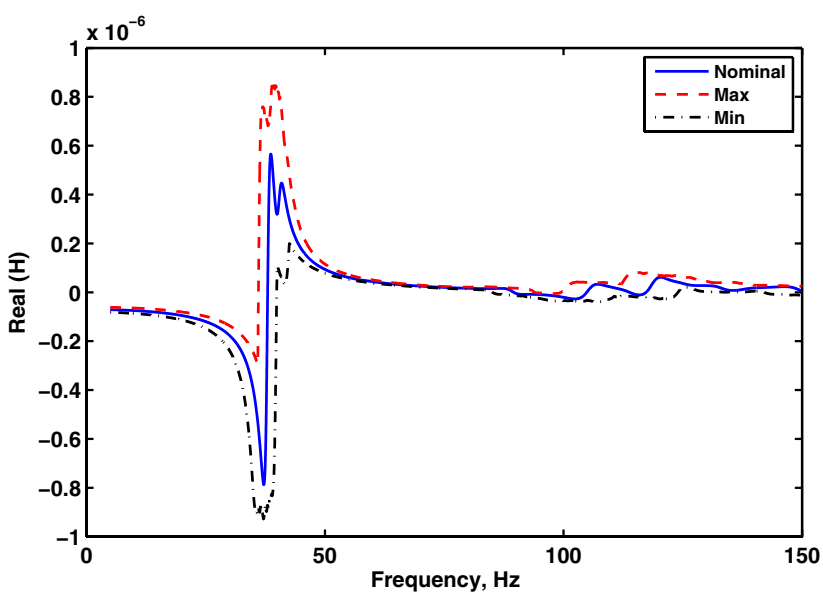

c) Location 3

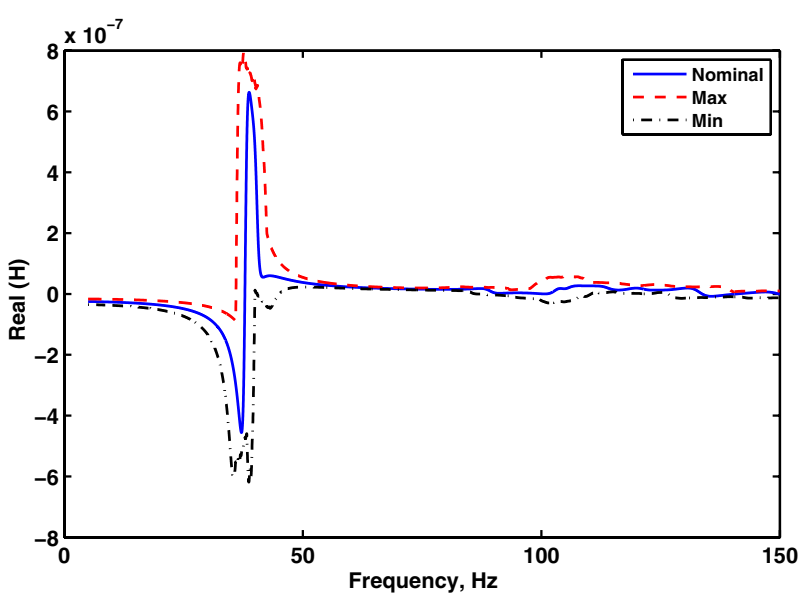

b) Location 2

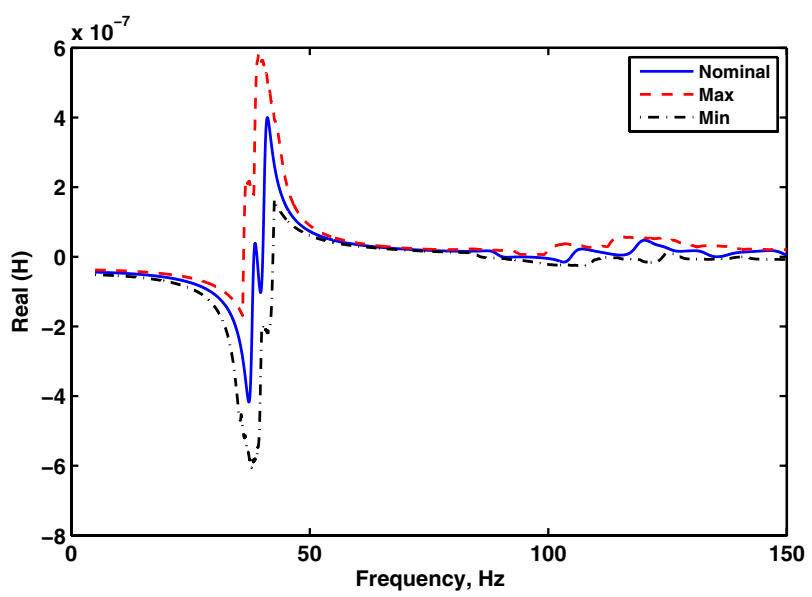

d) Location 4

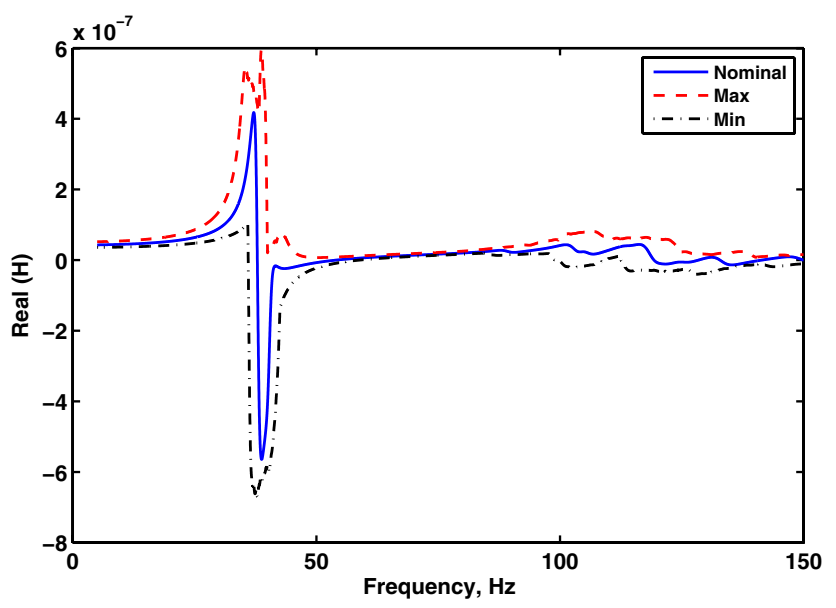

e) Location 5

Fig. 7 Frequency responses of Spacecraft 1.

response computation. To maintain the accuracy of the FRF, all modes in the required frequency band and sufficient modes in the lower and upper bands must be included in the computation of $H_{p q}(\Omega)$ [14]. In this study all the modes from 0 to $300 \mathrm{~Hz}$ were included to calculate the FRF in the frequency band of 5 to $150 \mathrm{~Hz}$ and obtained a very good comparison with MSC Nastran [25] results.

Modal-based displacement frequency responses at five different locations of Spacecrafts 1 and 2 for a unit-excitation force in $Z$ direction were computed using Eq. (15). Figure $\underline{6}$ shows the response and input locations in both spacecraft models. It was assumed that frequency and mode shape were corrupted, with 5\% inaccuracies and $2 \%$ modal damping. Maximum and minimum responses for each frequency step of $0.1 \mathrm{~Hz}$ were computed for the required frequency band of 5 to $150 \mathrm{~Hz}$. The average value of maximum (Max) and minimum (Min) response were computed based on 1000 analyses for each frequency step to get a statistical representation of the actual process. The real part of the FRF, Real $(\mathrm{H})$, for the different locations of Spacecraft 1, along with the nominal modal-displacement 
response function, is shown in the Fig 7. The nominal responses are calculated using the uncorrupted modal parameters along with the $2 \%$ modal damping.

Figure 8 shows the FRF of Spacecraft 2 for five different locations for a unit force at the base of the structure. Modal damping and errors in modal parameters were the same as in the case of Spacecraft 1. Tables 7 and 8 show the maximum and minimum responses in the frequency band (5 to $150 \mathrm{~Hz}$ ) based on an average result of 1000 analyses observed at five different locations of two spacecraft calculated using clean-and the noised-modal parameters. Spacecraft 1 has a considerably large deviation in peak response compared to the others. There is a maximum of $51.06 \%$ deviation in peak response when the modal parameters are corrupted with $5 \%$ error in

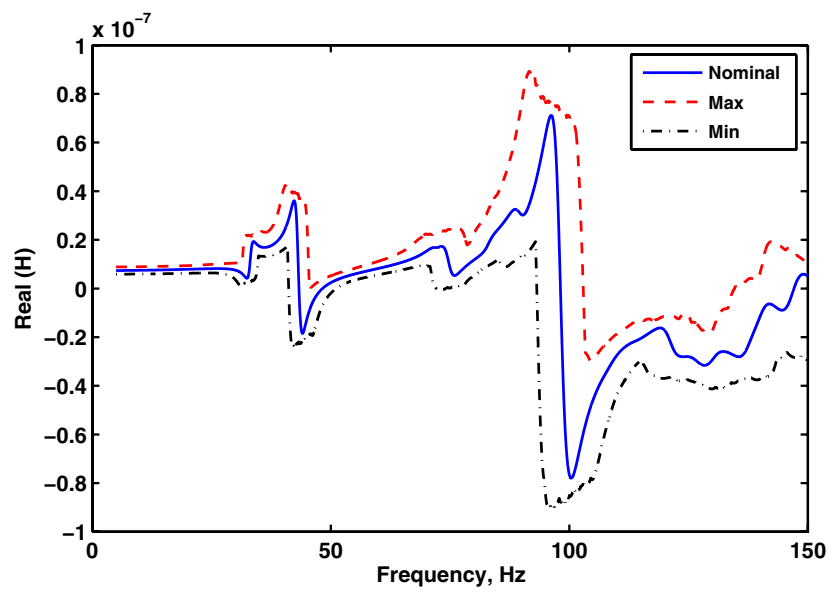

a) Location 1

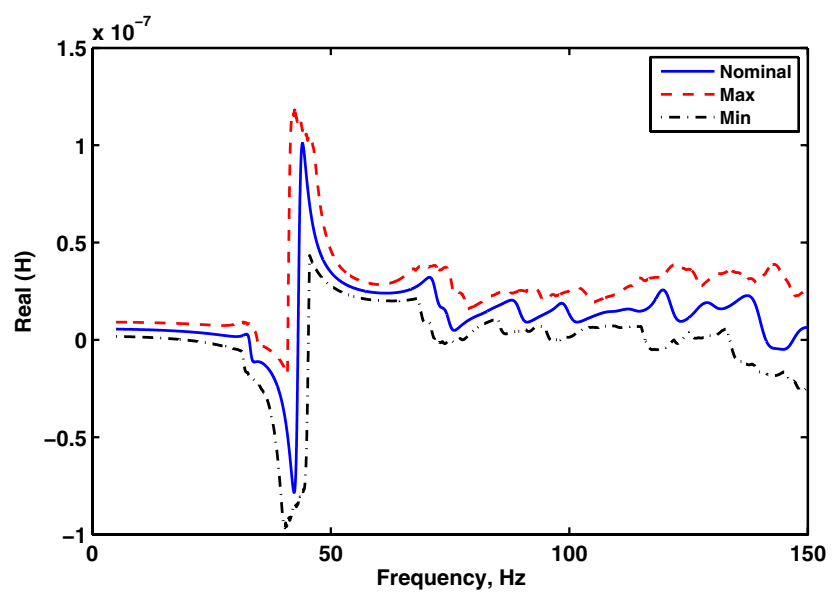

c) Location 3 comparison with nominal response. The estimation of minimum response also shows a change up to $51.4 \%$, as indicated in Table 8 . Spacecraft 2 shows only a maximum deviation of $25.42 \%$ in the peak frequency response and a maximum of $23.05 \%$ deviation in the minimum response. The average change in peak response for Spacecraft 1 is $36.25 \%$, whereas Spacecraft 2 has an average deviation of only $19.79 \%$. The average change in the minimum response for Spacecraft 1 is $33.87 \%$, but Spacecraft 2 has an average change of only $20.25 \%$. The previous results show that Spacecraft 2 is more vulnerable to inaccuracies. The results also found that Spacecraft 2 had more difficulty in passing the NCO compared to Spacecraft 1, but it was more robust in the prediction of frequency response when frequency and mode shapes were contaminated

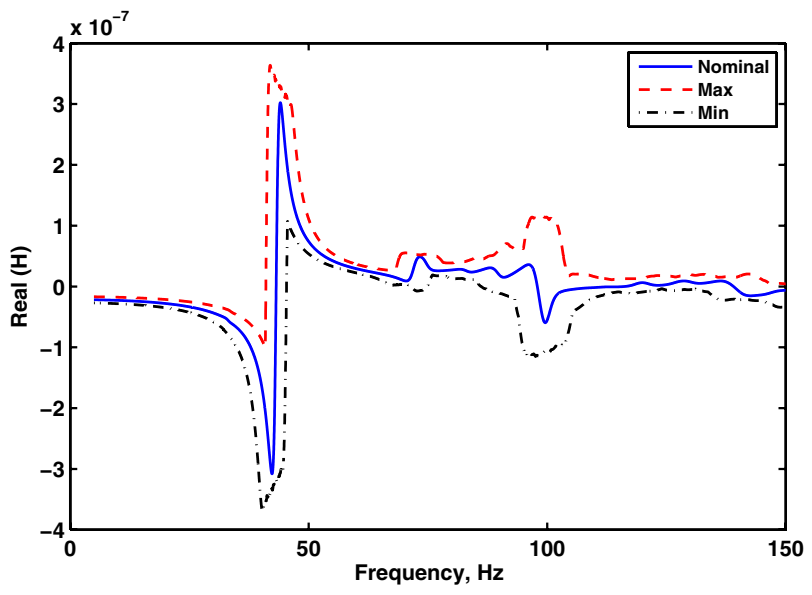

b) Location 2

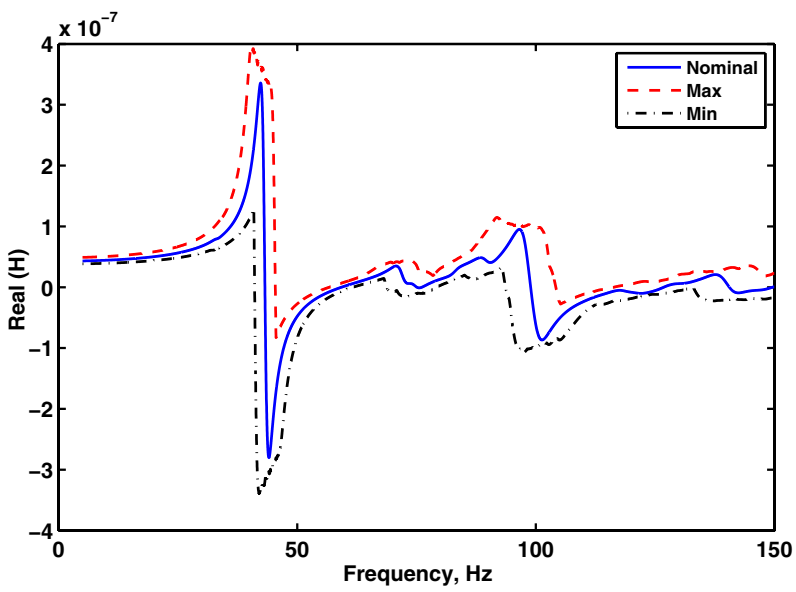

d) Location 4

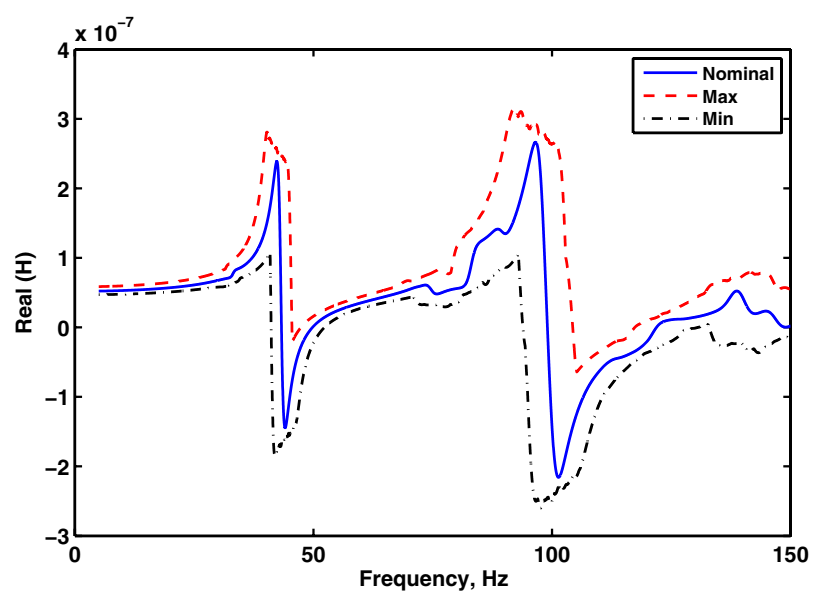

e) Location 5

Fig. 8 Frequency responses of Spacecraft 2. 
Table 7 Maximum frequency response

\begin{tabular}{lccccc}
\hline \hline \multicolumn{5}{c}{} & \multicolumn{5}{c}{ Peak response in the frequency range, real $(\mathrm{H})$} \\
\hline Structure & Location & Nominal modes & Inaccurate frequency and modes & Change, \% & Average change, \% \\
\hline Spacecraft 1 & 1 & $4.24 \times 10^{-7}$ & $5.17 \times 10^{-7}$ & 21.93 & \\
& 2 & $6.63 \times 10^{-7}$ & $7.95 \times 10^{-7}$ & 19.91 & 36.25 \\
& 3 & $5.66 \times 10^{-7}$ & $8.55 \times 10^{-7}$ & 51.06 & \\
& 4 & $4.00 \times 10^{-7}$ & $5.84 \times 10^{-7}$ & 46.00 & \\
& 5 & $4.18 \times 10^{-7}$ & $5.95 \times 10^{-7}$ & 42.34 & \\
Spacecraft 2 & 1 & $7.12 \times 10^{-8}$ & $8.93 \times 10^{-8}$ & 25.42 & \\
& 2 & $3.02 \times 10^{-7}$ & $3.64 \times 10^{-7}$ & 20.53 & \\
& 3 & $1.01 \times 10^{-7}$ & $1.18 \times 10^{-7}$ & 16.83 & \\
& 4 & $3.36 \times 10^{-7}$ & $3.97 \times 10^{-7}$ & 18.15 & \\
& 5 & $2.66 \times 10^{-7}$ & $3.14 \times 10^{-7}$ & 18.04 & \\
\hline \hline
\end{tabular}

Table 8 Minimum frequency response

\begin{tabular}{lccccc}
\hline \hline \multicolumn{6}{c}{ Minimum response in the frequency range, real $(H)$} \\
\hline Structure & Location & Nominal modes & Inaccurate frequency and modes & Change, \% & Average change, \% \\
\hline Spacecraft 1 & 1 & $-2.86 \times 10^{-7}$ & $-4.33 \times 10^{-7}$ & 51.40 & \\
& 2 & $-4.56 \times 10^{-7}$ & $-6.19 \times 10^{-7}$ & 35.75 & \\
& 3 & $-7.86 \times 10^{-7}$ & $-9.28 \times 10^{-7}$ & 18.07 & 33.87 \\
& 4 & $-4.17 \times 10^{-7}$ & $-6.06 \times 10^{-7}$ & 45.32 & \\
\hline Spacecraft 2 & 5 & $-5.64 \times 10^{-7}$ & $-6.70 \times 10^{-7}$ & 18.79 & \\
& 1 & $-7.79 \times 10^{-8}$ & $-9.11 \times 10^{-8}$ & 16.94 & \\
& 2 & $-3.08 \times 10^{-7}$ & $-3.69 \times 10^{-7}$ & 19.80 & \\
& 3 & $-7.85 \times 10^{-8}$ & $-9.66 \times 10^{-8}$ & 23.05 & \\
& 4 & $-2.80 \times 10^{-7}$ & $-3.39 \times 10^{-7}$ & 21.07 & \\
& 5 & $-2.16 \times 10^{-7}$ & $-2.60 \times 10^{-7}$ & 20.37 & \\
\hline \hline
\end{tabular}

with random inaccuracies. Hence, the ability to pass the NCO does not necessarily imply an accurate capability to predict the response.

\section{E. Selection of Optimum Sensor Locations Using SEREP Mass Matrix}

The selection of sensor locations from the available locations or DOFs can help to meet the quality criteria, such as NCO [18]. Effective independence matrix (EFI) is a proven technique to select the optimum sensor locations. This is carried out by calculating the EFI of the modes using Eq. (14) and eliminating the sensor locations corresponding to the least element in the diagonal of the EFI matrix. After eliminating the particular DOF, the EFI matrix must be recomputed, and then the procedure continues until the required number of sensor locations is left [19]. As per Eq. (13), SEREP reduced mass matrix, $\boldsymbol{M}_{\text {SEREP }}$ has a significant role in meeting the NCO criteria, and in turn, it is calculated using the mode shapes obtained using the different sensor locations. Hence, $\boldsymbol{M}_{\text {SEREP }}$ depends on the sensor locations. Using this property, SEREP mass matrix is employed to determine the optimum sensor locations, as in the case of EFI. The sensor location is iteratively removed for the location that corresponds to the least value in the diagonal of the $\boldsymbol{M}_{\text {SEREP }}$ matrix. Then the $\boldsymbol{M}_{\text {SEREP }}$ matrix is recomputed using the new sensor locations, and the procedure continues until the required number of sensor locations is reached. The procedure is illustrated in the flowchart shown in Fig. 9 , and it is applied to optimize the sensor locations of Spacecraft 2, as explained in the following section. A similar flowchart can be used for the EFI-based optimization by calculating the EFI matrix instead of $\boldsymbol{M}_{\text {SEREP }}$.

In the previous analysis, there were 99 DOFs used for the determination of 12 target modes for Spacecraft 2. For example, to select the 50 optimum sensor locations from the available 99 DOFs for the determination 12 target modes, the selection of sensor location was performed using three different methods. The obtained results are shown in Table 9. In the first method, 50 sensor locations were randomly selected from 99 locations, whereas the second and third methods used the procedure illustrated in the flowchart (Fig. 9) with the EFI and the $\boldsymbol{M}_{\text {SEREP }}$ matrices, respectively. There were 12 modes used in the computation of the $\boldsymbol{M}_{\mathrm{SEREP}}$ matrix. The probability of meeting the NCO for different percentages of inaccuracies was computed after the selection of optimum-sensor locations and performing 1000 trials for each noise level. The optimization was performed for three cases by considering different levels of

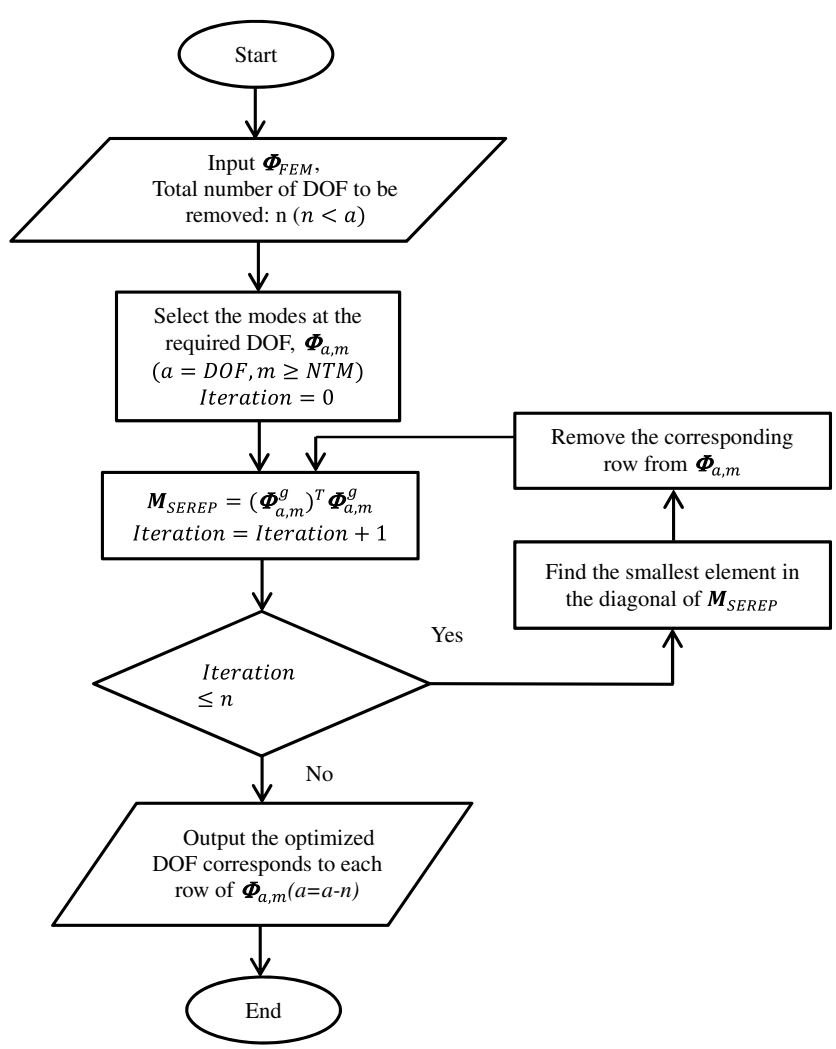

Fig. 9 Flowchart for sensor location optimization. 
Table 9 Optimization of sensor location for Spacecraft 2

\begin{tabular}{|c|c|c|c|c|c|c|c|c|c|}
\hline \multirow[t]{2}{*}{ DOF selection } & \multicolumn{8}{|c|}{ Percentage of inaccuracy } & \multirow[t]{2}{*}{ Case $(\mathrm{NTM}=12)$} \\
\hline & 50 & 25 & 10 & 5 & 2.5 & 1 & $0.1 \quad 0.01$ & 0.001 & \\
\hline Random & $\leftarrow$ & 0.0 & 6.5 & 72.5 & 100.0 & $\rightarrow$ & & & Error in experimental modes \\
\hline EFI & $\leftarrow$ & 0.0 & 66.0 & 100.0 & $\rightarrow$ & & & & \\
\hline SEREP & 0.0 & 0.2 & 75.3 & 100.0 & $\rightarrow$ & & & & \\
\hline Random & $\leftarrow$ & 0.0 & 6.1 & 75.3 & 100.0 & $\rightarrow$ & & & Error in FE modes \\
\hline EFI & $\leftarrow$ & 0.0 & 11.0 & 98.6 & 100.0 & $\rightarrow$ & & & \\
\hline SEREP & $\leftarrow$ & 0.0 & 25.3 & 100.0 & $\rightarrow$ & & & & \\
\hline Random & $\leftarrow$ & 0.0 & 0.3 & 37.5 & 96.4 & 100.0 & $\rightarrow$ & & Error in both FE and experimental modes \\
\hline EFI & $\leftarrow$ & 0.0 & 3.4 & 86.2 & 100.0 & $\rightarrow$ & & & \\
\hline SEREF & $\leftarrow$ & 0.0 & 9.1 & 94.2 & 100.0 & $\rightarrow$ & & & \\
\hline
\end{tabular}

inaccuracies in the experimental modes, the FE modes, and in both the experimental and FE modes.

When sensor locations are randomly selected, even $10 \%$ error in the experimental modes leads to a very low percentage (6.5) of passing the NCO criteria, as shown in Table 9. If an optimization algorithm is used to choose the location, it can boost the success percentage to 66 and $75.3 \%$ for EFI and SEREP-based optimization, respectively. When the inaccuracy is present in the experimental mode shapes, both optimization procedures can give better results compared to the case when inaccuracy is present only in the FE modes because the FE error is directly affecting the computation of the EFI and $\boldsymbol{M}_{\text {SEREP }}$ matrices. Nevertheless, for all the cases of inaccuracies, both EFI and SEREP-based sensor-location optimization leads to a higher probability of meeting the NCO criteria, but the SEREP-based optimization always yields better results than the EFI-based optimization. The sensor-location optimization for the other spacecraft models also indicated that the SEREP-based optimization is better than the EFI-based optimization in meeting the NCO criteria.

\section{Conclusions}

The robustness of system equivalent reduction expansion process (SEREP)-based test-analysis model (TAM) with respect to inaccuracies in experimental and analytical mode shapes has been investigated with the help of real spacecraft-structure finite-element models (FEMs). As expected, it has been observed that as the number of modes used in reduction to calculate the TAM matrix increases, the probability of successfully passing normalized cross-orthogonality (NCO) decreases. The analyses of three different spacecraft structures show that for practical purposes, the modes used in the SEREP-reduction process should only be the target modes. The computational efficiency of SEREPa deteriorates the test-analysis correlation to the extent that it leads to an unreasonable low probability of success, even when a very low level of inaccuracy $(0.1 \%)$ is present. The probability of successfully passing NCO depends not only on the amount of inaccuracy in the modes, but also on the relationship between the target modes. As the standard deviation of the diagonal terms of the effective-independence (EFI) matrix generated using the target modes increases, the probability of passing the NCO reduces. It has been observed that highly uncorrelated synthetic target modes generated using the random numbers have the highest probability of meeting the NCO requirement, and their EFI-matrix diagonals have the least standard deviation compared to other spacecraft modes. It was also shown with the help of spacecraft FEMs that, although a spacecraft FEM may fail in the NCO check using SEREP, it could still predict the displacement-based frequency-response function (FRF), which is proportional to stress and loads in structural members, with higher accuracy than the FEM that passes the NCO check. Hence, this type of modal-verification criterion does not assure a certain level of capability of the FEM to predict the FRF. A SEREP-based sensorlocation optimization is proposed to identify the optimal sensor locations, and it is found that the optimization yields better probability of passing the NCO than the EFI-based optimization.

\section{Acknowledgments}

The authors wish to acknowledge the Commonwealth Scholarship Commission, U.K. for their funding. They would also like to thank G. Richardson, Surrey Satellite Technology Limited, U.K., and A. Kiley, EADS Astrium Ltd, U.K. for providing the spacecraft FEM for this study, as well as S. Redi, Astronautics Research Group, University of Southampton for the computer-programming support.

\section{References}

[1] Allemang, R. J., "The Modal Assurance Criterion: Twenty Years of Use and Abuse," Sound and Vibration, Vol. 37, No. 8, 2003, pp. 14-23.

[2] Wijker, J., Mechanical Vibrations in Spacecraft Design, 1st ed., Springer, Berlin, 2004, pp. 343-367.

[3] Kammer, D. C., "Test Analysis Model Development Using an Exact Modal Reduction," International Journal of Analytical and Experimental Modal Analysis, Vol. 2, No. 4, 1987, pp. 174-179.

[4] Guyan, R. J., "Reduction of Mass and Stiffness Matrices," AIAA Journal, Vol. 3, No. 2, 1965, p. 380. doi: $10.2514 / 3.2874$

[5] Irons, B., "Structural Eigenvalue Problems: Elimination of Unwanted Variables," AIAA Journal, Vol. 3, No. 5, 1964, pp. 961-962.

[6] Kidder, R. L., "Reduction of Structural Frequency Equations," AIAA Journal, Vol. 11, No. 6, 1973, pp. 892-892. doi: $10.2514 / 3.6852$

[7] Paz, M., "Dynamic Condensation," AIAA Journal, Vol. 22, No. 5, 1984 , pp. 724-726. doi: $10.2514 / 3.48498$

[8] Kammer, D. C., "A Hybrid Approach to Test Analysis Model Development for Large Space Structures," Journal of Vibration and Acoustics-Transactions of the ASME, Vol. 113, No. 3, 1991, pp. 325332.

[9] O'Callahan, J., "A Procedure for an Improved Reduced System (Irs) Model," Proceedings of the 7th International Modal Analysis Conference, Las Vegas, Jan. 1989, pp. 17-21.

[10] Friswell, M. I., Garvey, S. D., and Penny, J. E. T., "Model Reduction Using Dynamic and Iterated Irs Techniques," Journal of Sound and Vibration, Vol. 186, No. 2, 1995, pp. 311-323. doi:10.1006/isvi.1995.0451

[11] Berman, A., and Nagy, E. J., "Improvement of a Large Analytical Model Using Test Data," AIAA Journal, Vol. 21, No. 8, 1983, pp. 1168 1173. doi: $10.2514 / 3.60140$

[12] Kabe, A. M., "Stiffness Matrix Adjustment Using Mode Data," AIAA Journal, Vol. 23, No. 9, 1985, pp. 1431-1436. doi: $10.2514 / 3.9103$

[13] Butland, A., and Avitabile, P., "A Reduced Order, Test-Verified Component Mode Synthesis Approach for System Modeling Applications," Mechanical Systems and Signal Processing, Vol. 24, No. 4, 2010, pp. 904-921. doi:10.1016/j.ymssp.2009.08.008

[14] Nimityongskul, S., and Kammer, D. C., "Frequency Domain Model Reduction Based on Principal Component Analysis," Mechanical Systems and Signal Processing, Vol. 24, No. 1, 2010, pp. 41-51. doi:10.1016/j.ymssp.2009.05.005

[15] Bergman, E. J., Allen, M. S., Kammer, D. C., and Mayes, R. L., "Probabilistic Investigation of Sensitivities of Advanced Test-Analysis Model Correlation Methods," Journal of Sound and Vibration, Vol. 329, No. 13, 2010, pp. 2516-2531. doi:10.1016/j.jsv.2010.01.009 
[16] O'Callahan, J., Avitabile, P., and Riemer, R., "System Equivalent Reduction Expansion Process (SEREP), " Proceedings of the 7th International Modal Analysis Conference, Las Vegas, Jan. 1989, pp. 29-37.

[17] Avitabile, P., "Some Common Issues in Modal Correlation/Validation," Proceedings of the 24th International Modal Analysis Conference, St. Louis, MO, Jan. 2006.

[18] Aglietti, G. S., Walker, S. J. I., and Kiley, A., 'On the Use of 'SEREP' for Satellite FEM Validation," Proceedings of the 4th International Conference on Advanced Computational Engineering and Experimenting, Paris, July 2010.

[19] Aglietti, G. S., Redi, S., Walker, S. J. I., and Kiley, A., "Robustness of the Orthogonality Checks on a Satellite Fem Using a Serep Test Analysis Model," Proceedings of the 24th International Conference on Noise and Vibration Engineering and 3rd International Conference on Uncertainty in Structural Dynamics, Leuven, Belgium, Sep. 2010.

[20] "Loads Analysis of Spacecraft and Payloads," NASA-STD-5002, https:// standards.nasa.gov/training/nasa-std-5002/index.html [retrieved 23 March 2011].

[21] "Modal Survey Assessment," ECSS-E-ST-32-11C, European Space Agency, July 2008, p. 49.

[22] Craig, R. R., and Bampton, M. C., "Coupling of Substructures for Dynamic Analyses," AIAA Journal, Vol. 6, No. 7, 1968, pp. 1313-1319. doi: $10.2514 / 3.4741$
[23] Avitabile, P., "Model Reduction and Model Expansion and Their Applications Part I: Theory," Proceedings of the 23rd International modal Analysis Conference, Orlando, FL, Jan. 2005.

[24] Penrose, R., "A Generalised Inverse for Matrices," Mathematical Proceedings of the Cambridge Philosophical Society, Vol. 51, No. 03 , 1955, pp. 406-413. doi: $10.1017 / \mathrm{S} 0305004100030401$

[25] "MSC Nastran Users Manual," MSC Software Corporation, CA, 2001

[26] Chung, Y. T., and Sernaker, M. L., "Assessment of Target-Mode Selection Criteria for Payload Modal Survey," Proceedings of the 12th International Modal Analysis Conference, Honolulu, HI, 1994, pp. 272-279.

[27] Kammer, D. C., "Effect of Noise on Sensor Placement for On-Orbit Modal Identification of Large Space Structures," Journal of Dynamic Systems Measurement and Control-Transactions of the ASME, Vol. 114, No. 3, 1992, pp. 436-443.

[28] Friswell, M. I., and Mottershead, J. E., "Finite Element Modelling," Finite Element Model Updating in Structural Dynamics, edited by G. M. L. Gladwell, Vol. 38, Kluwer Academic Publishers, Dordrecht, The Netherlands, 1995, pp. 7-35. 